Revue internationale de l'économie sociale

Recma

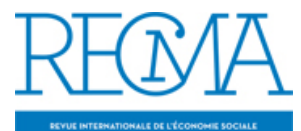

\title{
L'économie sociale et solidaire au Mexique : évolution historique et perspectives
}

\section{Francisco Ramirez-Mendez}

Numéro 335, janvier 2015

URI : https://id.erudit.org/iderudit/1028536ar

DOI : https://doi.org/10.7202/1028536ar

Aller au sommaire du numéro

\section{Éditeur(s)}

Association Recma

\section{ISSN}

1626-1682 (imprimé)

2261-2599 (numérique)

Découvrir la revue

\section{Citer cet article}

Ramirez-Mendez, F. (2015). L'économie sociale et solidaire au Mexique :

évolution historique et perspectives. Revue internationale de l'économie sociale, (335), 103-110. https://doi.org/10.7202/1028536ar 


\title{
L'ÉCONOMIE SOCIALE ET SOLIDAIRE AU MEXIQUE : ÉVOLUTION HISTORIQUE ET PERSPECTIVES
}

\author{
par Francisco Ramirez-Mendez*
}

\footnotetext{
* Doctorant au Centre de recherche sur l'intégration économique et financière (Crief) de l'université de Poitiers.

Cet article a bénéficié des conseils et des orientations de mon directeur de thèse, Gilles Caire. Je l'en remercie chaleureusement (NdA).
}

L’ESS présente au Mexique des aspects spécifiques résultant d'une relation complexe entre la réalité socioéconomique du pays, fruit de son histoire, et les politiques publiques qui tendent à agir en fonction des intérêts conjoncturels ou politiques définis par le modèle néolibéral en vigueur.

Le processus historique de construction de l'ESS au Mexique peut se diviser en cinq phases, correspondant à des dynamiques qui interfèrent souvent.

\section{LES ORIGINES DE L'ESS AU MEXIQUE, DE LA PÉRIODE PRÉ-HISPANIQUE AU XIX SIÈCLE}

Dans de nombreuses communautés indigènes, comme les Calpulli Mexicas, le conseil des anciens coordonnait les activités et octroyait les terres aux familles, en désignant celles qui devaient être travaillées collectivement. De plus, certains travaux généraux devaient être réalisés pour toute la population (temples, greniers, magasins, etc.) et des réserves de nourriture étaient constituées afin de pouvoir faire face aux périodes de sécheresse, de gel ou de guerre et de venir en aide aux personnes inaptes au travail physique. Après la conquête militaire espagnole et durant la période coloniale, la pratique s'est étendue aux magasins communaux, où les indigènes déposaient le produit de leurs récoltes en prévision des temps difficiles. Officiellement, le coopérativisme apparaît au Mexique en 1839, soit moins de deux décennies après l'indépendance (1821), avec la fondation à Orizaba de la première caisse d'épargne pour les employés et les artisans. Celle-ci présente alors toutes les caractéristiques coopératives et mutualistes inspirées par les socialistes utopiques français (Izquierdo, 2009). En 1873 est créé le premier atelier coopératif, avec en son sein la première société coopérative, qui rassemble vingt-six tailleurs. La même année, la première coopérative de consommation des ouvriers et des colons voit le jour, dans le quartier Buenavista de la ville de Mexico (Velazquez, 2013). A la suite, sont également créées une « société progressiste de charpentiers » et d'autres coopératives de production de chapeaux (Izquierdo, 2009). Ce premier mouvement coopératif et social trouve un relais auprès du Congrès général ouvrier de 1876, qui décide de promouvoir les coopératives. Le Code du commerce de 1889-1890 considère ces dernières comme une variante des sociétés commerciales, en leur accordant un intérêt certes inédit, mais néanmoins très limité (Fritz, 1986). 


\section{LA CONSOLIDATION DE L'ÉCONOMIE SOCIALE APRĖS LA RÉVOLUTION MEXICAINE}

La révolution mexicaine (1910-1920) présente deux aspects intéressants pour l'analyse de l'ESS: d'un côté, l'essor d'un militantisme ouvrier plus ou moins d'inspiration coopérativiste ${ }^{(1)}$ et, d'un autre, la structuration du modèle de l'ejido, qui - à notre sens - est le meilleur exemple d'un modèle propre à l'ESS au Mexique, comme nous le verrons plus loin.

(1) Il existait alors un Parti national coopérativiste, dont le président, Emilio Portes Gil, fut par la suite président du Mexique de 1928 à 1930.

Lactivisme ouvrier post-révolutionnaire aboutit en 1927 au vote de la première loi générale sur les sociétés coopératives, qui sera modifiée en 1933. Dans ce contexte de développement du mouvement coopératif, est créée en 1931 la coopérative la plus emblématique du Mexique, la Cruz azul (Croix bleue), suivie quelques années plus tard de la coopérative Excelsior, avec deux cent cinquante associés, et d'une autre coopérative, celle des Aiguiseurs de Tampico.

En 1938, l'essor du mouvement coopératif mexicain est favorisé par une loi adoptée par le gouvernement du général révolutionnaire Lázaro Cárdenas, célèbre pour avoir réalisé de grandes réformes sociales et nationalisé l'industrie pétrolière. Cette loi est la première à établir clairement que seuls les travailleurs peuvent participer à des coopératives et définit certains des principes fondamentaux de coopération, notamment le principe d'égalité des devoirs et des droits pour les coopérateurs, un vote par associé, l'absence de but lucratif pour les entreprises coopératives et la possibilité pour les syndicats de travailleurs d'organiser des coopératives de consommation (Izquierdo, 2009).

Ensuite, à partir des années 50, sont fondées les principales caisses populaires d'épargne. Les années 70 sont marquées par la multiplication des coopératives de pêcheurs, qui se voient octroyer le monopole de l'exploitation des espèces marines de plus grande valeur commerciale. Parmi d'autres mesures, signalons un programme de promotion des coopératives scolaires (Fritz, 1986).

\section{L'INTÉGRATION DE LA DYNAMIQUE EJIDALE DANS LE SYSTĖME COOPÉRATIF MEXICAINAU COURS DES ANNÉES7O}

Durant la décennie 1970, grâce au contrôle absolu exercé par le Parti révolutionnaire institutionnel (PRI) sur l'Etat mexicain, les coopératives s'intègrent dans la logique étatique, d'une part, en venant en appui des actions de l'Etat et, d'autre part, en adoptant des pratiques bureaucratiques qui les amènent à rompre avec certains de leurs principes fondateurs. Du fait de son inclusion dans ces logiques bureaucratiques et du clientélisme politique, le mouvement coopératif se trouve quelque peu dénaturé (Velasquez, 2013).

Cependant, de 1973 à 1983, le nombre de coopératives a considérablement augmenté, en raison du développement de coopératives de participation de l'Etat, qui en trois ans ont permis de créer 2032 coopératives de production. En revanche, l'impact social général des coopératives reste faible, puisqu'elles n'emploient que $1,1 \%$ de la population active et ne concernent que $0,7 \%$ de la population mexicaine (Fritz, 1986). 


\section{LA CRISE DES COOPÉRATIVES DANS LE CONTEXTE NÉOLIBÉRAL DOMINANT}

La mise en marche du modèle néolibéral à partir de 1982 affecte significativement le mouvement coopératif. Elle se traduit, au milieu des années 80, par la suppression par le gouvernement de la Direction générale de promotion de la coopération et de la Banque nationale d'appui aux coopératives, qui existaient depuis le gouvernement du général Cárdenas (1936-1940).

Ce fait, combiné avec la tendance ultralibérale de l'économie et les crises économiques subséquentes avec des périodes d'inflation et de récession, provoque une grave crise interne au sein de ces coopératives peu efficaces, extrêmement bureaucratisées, sectorisées et sans véritable dynamique. La crise se solde en 1994 avec la modification de la loi coopérative, qui permet, entre autres mesures, l'association de personnes physiques et morales. L'inclusion des coopératives dans le registre public du commerce, en effaçant leur identité juridique propre, signe leur coup de grâce (Velazquez, 2013). Très peu ont survécu à ce processus.

\section{RÉSURGENCE DE L'ESS EN RÉPONSE AUX CONSÉQUENCES SOCIALES DU MODĖLE NÉOLIBÉRAL}

Cependant, d'autres options économiques ont été portées à travers le pays par divers mouvements de la société civile, affichant une identité ESS ou économie alternative. Ces initiatives, que l'on peut qualifier d'économie plurielle, selon la proposition de Jean-Louis Laville (Laville, Cattani, 2006), n’ont pas été élaborées dans le cadre d'un projet global ou national. Elles résultent de résistances populaires ou civiques - souvent locales ou sectorielles - aux politiques, ce qui leur confère certaines caractéristiques: elles sont généralement autonomes par rapport aux partis politiques ou aux grands mouvements sociaux (à l'exception du mouvement néo-zapatiste apparu sur la scène publique en 1994) et peu coordonnées entre elles. Bien que faisant preuve de flexibilité et d'esprit d'innovation, elles s'inspirent des expériences étrangères bien consolidées, comme l'économie sociale en France ou au Québec, les projets de buen vivir en Amérique du Sud, les activités de promotion et d’implication du Ciriec ou de Mondragón en Espagne.

\section{La nécessité d'une reconnaissance institutionnelle}

L'essor des initiatives de type ESS a exacerbéla nécessité d'une reconnaissance institutionnelle de ce modèle, dont la définition se révèle très complexe. La loi sur l'ESS, promulguée en mai 2012 et réformée en juin 2013, tente de cerner une réalité sociale (l'existence des coopératives et d'autres figures de l'ESS) sous l'influence des expériences européennes et canadienne, tout en l'ajustant au projet économique gouvernemental, auquel il confère une légitimité sociale, sans transformer fondamentalement sa politique d'orthodoxie économique. La loi vise l'article 25 de la Constitution mexicaine, qui concerne le «secteur social de l'économie». Ce concept, créé en 1983, "reconnaissait l'existence, dans le champ du système d'économie mixte du pays, d'un troisième secteur 
économique, composéd'organismes et d'entreprises de nature distincte de celles qui relèvent traditionnellement des secteurs public et privé».

Cependant, le texte juridique pêche par l'absence de définition concrète du concept de l'ESS, objet de la loi, alors qu'il décrit le fonctionnement du secteur et son intégration dans l'économie. Sans référence au concept de solidaire, il crée un organisme simplement nommé Institut national de l'économie sociale (Inaes).

D’un point de vue réglementaire, le secteur social de l'économie «fonctionne comme un système socioéconomique créé pour des organismes de propriété sociale fondés sur des relations de solidarité, de coopération et de réciprocité, privilégiant le travail et l'être humain, structurés et administrés sous une forme associative, pour satisfaire les besoins de leurs adhérents et des communautés dans lesquelles ils se développent». Ce secteur est composé des entités suivantes: ejidos (voir infra), communautés, organisations de travailleurs, sociétés coopératives, entreprises appartenant majoritairement ou exclusivement aux travailleurs et toutes les formes d'organisation sociale pour la production, la distribution et la consommation de biens et de services socialement nécessaires.

Ce critère légal a servi de base à l'élaboration d'un premier diagnostic national, réalisé par l'Inaes en coordination avec l'université ibéro-américaine Puebla (tableau 1).

Toutefois, une étude réalisée par Carlos Heredia et Adriana Gómez rapporte qu'«il existe environ 47000 entreprises associatives solidaires, avec huit millions d'associés, ce qui équivaut à $18 \%$ de la population active», et selon cette étude, "en 2008, [...] il y avait 9767 coopératives, qui employaient 182928 personnes. Les coopératives mexicaines représentaient, respectivement, 0,13\% et 0,25\% du PIB de cette année-là. Il y a, en outre, environ 5,6 millions de membres des ejidos et des communautés dans les zones rurales au Mexique» (Heredia, 2013, p. 7).

Selon d'autres études, "approximativement $90 \%$ des coopératives étudiées se sont constituées entre 1995 et 2002 »(Rojas Herrera, p. 127). En 2011, le ministre des Finances mentionnait même que 8,9 millions de personnes travaillaient dans le secteur social de l'économie (Campos in Oulhaj, Saucedo, 2013).

\section{Tableau 1}

\begin{tabular}{|l|r|}
\hline Organismes du secteur de l'économie sociale & Nombre estimé en 2014 \\
\hline Ejidos & 29555 \\
\hline Sociétés de production rurale & 9471 \\
\hline Unions de ejidos & 6426 \\
\hline Coopératives de production et de consommation & 3059 \\
\hline Communautés & 1744 \\
\hline Sociétés de solidarité sociale & 1680 \\
\hline Unions de crédit & 1540 \\
\hline Caisses d'épargne de travailleurs & 60943 \\
\hline Total des organismes du secteur de l'économie sociale & 2359 \\
\hline
\end{tabular}

Source: UIA, 2013, p. 23. 
Il est donc difficile de faire un vrai diagnostic sur l'ESS au Mexique, car « $i l$ [n'y] existe pas de mesures précises de l'ESS comme dans d'autres pays. Il n'y a pas un dégroupement du PIB mexicain qui permettrait de connaître la taille du secteur social de l'économie dans le détail » (Heredia, 2013, p. 6).

A tous les niveaux de l'Etat, on note une volonté permanente d'institutionnaliser les différents acteurs de l'ESS, soit en les intégrant à l'économie globale, soit en les impliquant dans les schémas du clientélisme politique. Chaque programme de financement officiel s'inscrit en effet dans ce que les chercheurs, les universitaires, les opposants et les ONG dénoncent comme l'une des stratégies les plus communes du vote clientéliste au Mexique.

Cette politique se heurte au processus social et civique de l'ESS qui, en dépit des efforts déployés au niveau institutionnel pour la reconfigurer, évolue de façon propre.

\section{Un secteur hétérogène}

Sans prétendre offrir un panorama exhaustif de l'ESS, le tableau 2 vise à illustrer la diversité actuelle et les racines spécifiques de ce secteur au Mexique. Il n'existe pas de structure fédérative et de coordination, mais des initiatives ont été lancées en vue de regrouper et de clarifier les concepts et de définir des stratégies communes. C'est ce qu'a tenté de faire l'Espace d'économie solidaire (EcoSol), en réalisant il y a dix ans trois ateliers nationaux qui ont publié une "charte d'engagement en faveur de la création d'une économie juste et fraternelle».

Le modèle le plus caractéristique de l'ESS au Mexique est l'ejido, qui, pour de nombreux anthropologues ou ethnologues, tire son origine directe des structures préhispaniques du Calpulli ou Chinancalli. Il a été réglementé après la révolution mexicaine (1910-1920) en réponse aux demandes du zapatisme (groupe révolutionnaire dirigé par Emiliano Zapata, porteur de fortes revendications agraires et sociales). Sa structure juridique, très complexe, repose sur la dotation de terres (y compris bois et eaux) à destination d'un noyau de population converti en personne juridique de plein droit. Celuici obtient ainsi des droits collectifs ou individuels sur les terres qu'il peut travailler collectivement ou individuellement. La plus haute autorité juridiquement reconnue est l'assemblée ejidale, qui peut à son tour nommer les instances de gouvernance et de contrôle, comme le commissaire ejidal et le conseil de surveillance.

\section{Tableau 2}

\begin{tabular}{|l|l|}
\hline Type ESS & Acteur ESS \\
\hline Mouvements coopératifs & $\begin{array}{l}\text { Euskadi, union de coopératives Tosepan Titataniski (« Nous vaincrons } \\
\text { ensemble », en náhuatl), groupe Jade, union des producteurs biologiques } \\
\text { Beneficio Majomut, Maya Vinic de Chiapas }\end{array}$ \\
\hline $\begin{array}{l}\text { Coopératives de consommation } \\
\text { et de commerce équitable }\end{array}$ & $\begin{array}{l}\text { Commerce équitable Mexique, réseau de l'économie solidaire } \\
\text { Guadalajara et coopérative de consommation Micasa }\end{array}$ \\
\hline $\begin{array}{l}\text { Projets de mutualités } \\
\text { ou de banques coopératives }\end{array}$ & $\begin{array}{l}\text { Fonds Fides, caisse Morelia Valladolid, Conseil mexicain d'épargne } \\
\text { et de crédit populaire AC et Caisse populaire mexicaine }\end{array}$ \\
\hline
\end{tabular}


Il est remarquable que cette organisation autochtone, spécifiquement mexicaine, qui a obtenu sa reconnaissance juridique en 1915 au terme d'un processus historique particulier ait fini par pouvoir intégrer pleinement les concepts de l'ESS.

Les deux exemples les plus représentatifs du mouvement coopératif mexicain sont, d'une part, le cas de la coopérative Pascual et, d'autre part, celui de la coopérative Cruz azul. La coopérative Pascual était à l'origine une usine de fabrication de boissons gazeuses datant des années 50 qui, à la suite d'une grande grève syndicale (1982-1984), s'est transformée en coopérative, avec l'appui de différents mouvements de la société civile. Comptant plus de 5000 emplois directs, elle est aujourd'hui l'une des plus dynamiques entreprises mexicaines à l'échelle nationale (Marañon, 2013; Velazquez, 2013).

La coopérative Cruz azul, quant à elle, est une compagnie de ciment créée à la fin du XIX ${ }^{e}$ siècle, dans laquelle les travailleurs ont réussi à se syndicaliser grâce à une intervention directe du juge-conciliateur qui s'occupait légalement de leur cas. Cela leur a permis d'éviter des licenciements massifs et de créer la coopérative, comptant 192 travailleurs qui, avec l'appui des villages voisins, ont pu non seulement la faire fonctionner, mais aussi consolider une structure sociale coopérative autour du projet. Ils ont ainsi créé deux villes coopératives, un système éducatif, des cliniques, des centres culturels et même l'une des plus populaires et brillantes équipes de football professionnel du Mexique. En 2011, l'entreprise occupait le troisième rang du marché du ciment au Mexique et, selon son dernier rapport (2011), comptait 974 coopérateurs associés.

D'autres exemples méritent d'être mentionnés: les pratiques des communautés indigènes (reconnues comme acteurs de l'ESS dans les premières propositions de la loi), comme l'Union des communautés indigènes de la région de l'isthme et les divers mouvements indigènes au Chiapas, à Oaxaca, à Guerrero, notamment. L'essor des projets de monnaies alternatives au Mexique, malgré les menaces gouvernementales, est également très significatif. Celles-ci se développent aujourd'hui en systèmes d'économie solidaire d'un grand dynamisme.

\section{Un rapport conflictuel avec l'Etat}

La plupart de ces organisations ne sont pas nées d'initiatives gouvernementales ni d'actions citoyennes isolées ou philanthropiques, mais dans un contexte de confrontation avec le gouvernement mexicain. Une confrontation généralement non délibérée, mais qui s'est produite de facto. D’un point de vue général, se dégagent trois types de confrontation:

- historique, quand une forme d'ESS a dû être utilisée pour résoudre un problème social spécifique. L'ejido en est le meilleur exemple, car sa consolidation juridique a permis de canaliser le mécontentement paysan au Mexique. Les exemples de Pascual ou de Cruz azul sont également applicables;

- directe, de la figure de l'ESS avec l'Etat, soit parce que l'Etat ne la reconnaît pas légalement, soit parce qu'il nie sa légitimité ou encore parce que son existence entre en contradiction avec le système juridique mexicain. Appartiennent à cette catégorie les monnaies alternatives, les ONG des droits de l'homme et certaines organisations indigènes et sociales; 
- indirecte, quand les activités d'ESS gênent le fonctionnement de groupes corporatifs très corrompus qui génèrent inégalités sociales et pauvreté. Ces groupes combattent généralement les expériences d'ESS, grâce à des alliances politiques avec des agents gouvernementaux. Exemple: les activités coopératives et de commerce équitable face aux intermédiaires et aux groupes industriels et commerciaux.

Cette classification ne présente qu'un caractère illustratif et il existe bien d'autres acteurs ou activités impliqués dans d'autres types de confrontations. Tel est le cas des communautés indigènes historiquement engagées dans une résistance qui se cristallise souvent dans des formes d'ESS (coopératives, associations ou simplement modèles communautaires). Elles entrent alors en conflit soit avec l'Etat, qui cherche à les transformer en entreprises classiques ou à exercer sur elles un contrôle politique, soit avec des groupes d'intérêt (paramilitaires, gros propriétaires terriens, bûcherons illégaux Talamontes, narcotrafiquants). La confrontation est souvent violente, comme le montre l'actualité récente à Ayotzinapa, où des étudiants qui étaient en train de lever des fonds participatifs pour soutenir leur école ont été kidnappés par la police municipale, avec l'aide de l'armée mexicaine.

Enfin, il convient de signaler le très large mouvement associatif, qui n'est pas reconnu par le gouvernement mexicain comme faisant partie de l'ESS, au motif qu'il ne poursuit pas des fins "principalement économiques». Il n’en est pas moins l'un des piliers les plus solides des mouvements démocratiques au Mexique, bien que difficile à classer en raison de son hétérogénéité. Il n'existe aucune structure de coordination, mais un travail de liaison est réalisé par le réseau Convergences des organisations civiles pour la démocratie, auquel adhèrent principalement des associations de défense des droits de l'homme ou d'organisation civique, des projets de développement intégré, de communication sociale, etc.

\section{CONCLUSION}

Incontestablement très dynamique, l'ESS s'exprime au Mexique sous des formes diverses. Cependant, son évolution reste fortement liée au poids de la puissance publique. De manière paradoxale, la plupart des expériences positives se sont consolidées sur la base d'une opposition à l'Etat, allant parfois jusqu'à la confrontation. A l'exception du cas particulier de quelques ejidos et coopératives agricoles, presque tous les acteurs et mouvements de l'ESS (y compris les fondations) s'appuient sur une forte dynamique citoyenne. Il n'en demeure pas moins que, de manière constante, l'Etat joue un rôle très important dans la vie de l'ESS au Mexique, pour la demande de financements, de subventions ou simplement d'actions politiques favorisant le développement de ces acteurs.

L'ESS incarne actuellement l'un des plus grands espoirs de construction sociale et économique au Mexique et sa configuration sera déterminante pour le pays dans les années à venir. 


\section{BIBLIOGRAPHIE}

Fritz-Krockow B., 1986, "Evaluación del cooperativismo mexicano ", in Comercio exterior, vol. 36, n 9 , México.

Heredia Zubieta C., Chico Spamer A., 2013, Estudio de caso: el fondo Fides (México), México, centro Reliess.

Izquierdo Muciño M., 2009, "Problemas de las empresas cooperativas en México que atentan contra su naturaleza especial ", in Boletín de la Asociación internacional de derecho cooperativo, $\mathrm{n}^{\circ} 43-2009$, Bilbao.

Laville J.-L., Cattani A.D., 2006, Dictionnaire de l'autre économie, Saint-Amand, Gallimard. Oulhaj L., Saucedo Perez F., 2013, Miradas sobre la economía social y solidaria en México, Puebla, Centro internacional de investigación de la economía social y solidaria.

Marañon Pimentel B., 2013, La economía solidaria en México, México, IIE, Unam.
Rojas Herrera J.-J., 2013, « Panorama general del cooperativismo agropecuario en México ", in Estudios agrarios, Procuraduría agraria, México.

Universidad iberoamericana Puebla (UIA), secretaría de economía e Inaes, 2013, note de synthèse du diagnostic de programme de promotion de l'économie sociale.

Universidad iberoamericana Puebla, secretaría de economía e Inaes, 2013, diagnostic du programme de promotion de l'économie sociale.

Velazquez Garcia J.-M., 2013, El movimiento cooperativo en México: la búsqueda de alternativas al desarrollo social, 2000-2010, México,Movimientociudadano, Partido político nacional.

A RETROUVER DANS LES ARCHIVES DE LA RECMA (RECMA.ORG/ARTICLES)

Touzard J.-M., Vandame R., « La coopérative comme outil du développement durable: le cas des coopératives d'apiculteurs au Mexique et au Guatemala », Recma, n³13, 2009.

Hostelet G., « Le Mexique d'aujourd’hui », Recma, n 55, 1935.

Léon C., "Les coopératives scolaires au Mexique », Recma, nº 48, 1933. 